\title{
Effect of self-triage on waiting times at a walk-in sexual health clinic
}

\author{
Samantha Hitchings, Janet Barter
}

\begin{abstract}
Background and methodology Lengthy waiting times can be a major problem in walk-in sexual health clinics. They are stressful for both patients and staff and may lead to clients with significant health issues leaving the department before being seen by a clinician. A self-triage system may help reduce waiting times and duplication of work, improve patient pathways and decrease wasted visits. This paper describes implementation of a self-triage system in two busy sexual and reproductive health clinics. Patients were asked to complete a self-assessment form on registration to determine the reason for attendance. This then enabled patients to be directed to the most appropriate specialist or clinical service. The benefits of this approach were determined by measuring patient waiting times, reduction in unnecessary specialist review together with patient acceptability as tested by a patient satisfaction survey. The ease of comprehension of the triage form was also assessed by an independent readers' panel.
\end{abstract}

Results A total of 193 patients were recruited over a 4-month period from November 2004 to February 2005. Patients from the November and December clinics were assigned to the 'traditional treatment' arm, with patients at subsequent clinics being assigned to the 'self-triage' system. Waiting times were collected by the receptionist and clinic staff. Ninety six patients followed the traditional route, 97 the new self-triage system. Sixty-nine (35.8\%) patients completed the satisfaction survey. The self-triage system significantly reduced waiting time from $40(22,60)$ to $23(10,40)$ minutes [results expressed as median (interquartile range)]. There was a non-significant reduction in the proportion of patients seeing two clinicians from $21 \%$ to $13 \%(p=0.17)$. Satisfaction levels were not significantly altered (95\% compared to $97 \%$ satisfied, $p=0.64)$. The readers' panel found the triage form both easy to understand and to complete.

Conclusions Self-triage can effectively reduce clinic waiting times and allow better organisation of resources. Urgent cases can be prioritised. This process appears to be acceptable to and understandable by patients.

Keywords clinic waiting times, patient satisfaction, selftriage, sexual heath clinic, walk-in clinic

J Fam Pann Reprod Health Care 2009; 35(4): 227-231

(Accepted 27 August 2009)

\section{Introduction}

Sexual health care is deemed by the Department of Health to be a key priority for the National Health Service (NHS) In November 2004, the Public Health White Paper 'Choosing Health' stressed the importance of modernising NHS sexual health services with an emphasis on easy access to testing and treatment of sexually transmitted infections (STIs). ${ }^{1}$

Southwark, in South London, is an area with one of the highest prevalences of STIs, teenage pregnancy and abortion in the UK. ${ }^{2}$ In 2004, when this study was initiated, the age-standardised abortion rate in Southwark was 46 per 1000 women, compared with 17.8 per 1000 for England and Wales. ${ }^{2}$ In order to improve access to STI health care, family planning clinics in Southwark also began testing and treatment of common genital infections. This has led to a significant increase in workload, creating a challenge in managing these patients without reducing the quality of care received by those attending clinics for contraception. During 2003-2004, the total number of clinical consultations increased by $24 \%$ (from 15370 to 19051 ).

For many years these clinics had been operating a walkin, open-access system, which was thought to best meet the needs of the local population. The increasing demand, however, had an impact on the waiting time in the clinics, with increasing patient dissatisfaction.

\section{Department of Reproductive and Sexual Health, Enfield Primary} Care Trust, Enfield, UK

Samantha Hitchings, MRCOG, MFSRH, Specialist Registrar

Janet Barter, FRCOG, FFSRH, Consultant

Correspondence to: Dr Samantha Hitchings, Town Clinic,

33 Eaton Road, Enfield, Middlesex EN1 1NJ, UK.

E-mail: samantha.hitchings@enfield.nhs.uk
Key message points

- Self-triage assessment forms effectively reduce clinic waiting times, improving patient pathways and use of resources.

- Triage with this system is acceptable to patients as judged by patients and a readers' panel.

Southwark and Lambeth sexual health departments were chosen by the Guy's and St Thomas's modernisation initiative $^{3}$ to take part in a modernisation programme to improve patients' access to clinics and overall experience. ${ }^{4}$ The first step in examining the service was to 'map' the patient pathway from their initial contact with the clinic to when they left it. ${ }^{5}$ Process mapping identifies potential problems from the patient's perspective and helps the development of improvements. The process mapping exercise identified long waiting times in clinic and the unnecessary requirement to be seen by more than one health care professional as key concerns. The patient's experience was also reflected by the beliefs of the clinic staff, who felt that waiting times could be excessive. In some instances, for example, patients were turned away after waiting for 2 hours or more because an intrauterine device (IUD) could not be fitted at the end of a clinic. The clinic staff also felt that it was difficult to organise a clinic if the reason for patients' attendance was unknown. They felt that if they understood the reason for each patient's attendance then they could direct them to the most appropriate clinician or alternative service. This would lead to reduced duplication of work, as a patient would be less likely to be questioned or examined twice and would not have wait a second time between clinicians. They could also ensure that those who needed to be seen urgently were prioritised. 


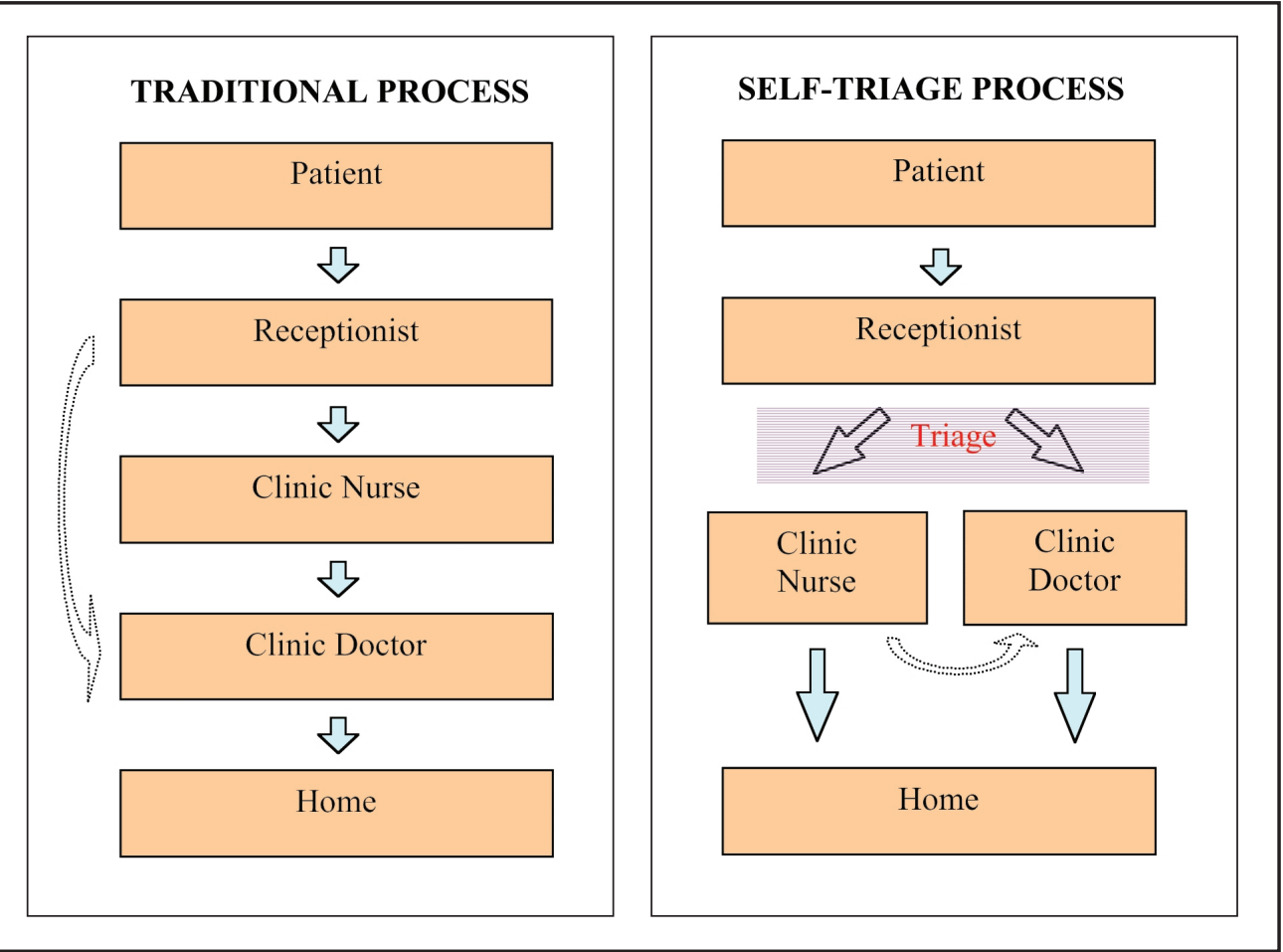

Figure 1 Traditional and self-triage pathways (major pathway: solid arrow; minor flow: dashed arrow)

One suggested method to help patient flow was a selfassessment triage form, completed by the patient at reception to indicate the reason for attendance. This form could enable a receptionist to direct each patient to a doctor or a nurse, as appropriate, or to another more appropriate clinic. It was also felt that a written triage method at reception was more confidential and would involve less staff time than verbal triage.

There is no published literature regarding the effect of triage on waiting time in family planning clinics. The triage process, however, has long been used in medicine as a method of prioritising patients when medical resources are limited, and is increasingly used in genitourinary medicine (GUM) clinics where demands on services are increasing, owing to the government's 48-hour waitingtime target.

Griffiths et al. evaluated a policy of triage in walk-in GUM clinics. ${ }^{6}$ They triaged patients according to age, symptoms and history of exposure to STIs. They found that the incidence of sentinel STIs (chlamydia and gonorrhoea) was significantly higher in those individuals who satisfied the triage criteria than in those who did not $(15.8 \%$ vs $1.7 \%$ ). The application of their triage method helped ensure that access was available to those who required more immediate attention.

Triage by specialist nurses within GUM clinics has been shown to provide an effective and high-quality service to patients with $95 \%$ compliance to clinical guidelines. ${ }^{7}$ Knight and McNulty reported nurse-led triage, directing a patient to the most appropriate clinician, could halve waiting times (from 24 to 12 minutes).$^{8}$ In general, clinic staff felt that triage improved patient flow and reduced patient anxiety and aggression. ${ }^{8}$

This article reports the first study of the use of a selfassessment triage form in two busy sexual and reproductive health (SRH) clinics. We evaluated patient clinic waiting times before and after the system was introduced, the number of clinicians seen by a patient and the total treatment time. An assessment of the acceptability of the method to patients was made together with an evaluation of the triage form by a readers' panel.

\section{Methods \\ Study design}

This was a prospective study that took place in two SRH clinics located in Southwark, South London, UK during 2004 and 2005. All patients in seven clinical sessions immediately before the introduction of the self-assessment triage form (traditional system) and in six clinics after selftriage were assessed in order to collect equal numbers of patients.

\section{Traditional system}

In the traditional clinic system, a patient entering clinic attended reception where they were registered and their notes handed to the clinic nurse. The nurse saw most patients first, managing the patient himself/herself if possible. If necessary, the patient would then be transferred to the doctor. Occasionally, the doctor would not be able to manage the problem and would have to redirect the patient to another clinic (Figure 1).

\section{Self-triage system}

A simple self-assessment triage form (Figure 2) was given to every patient attending reception. The receptionist made it clear that the form was confidential and would only be used by the clinic staff. The patient was asked to fill out the form at the reception desk, or in the waiting area, at the same time as a standard registration form. The triage form listed common presenting conditions from which the patient could choose to indicate the reason for their attendance. If, however, none of these options was applicable, the patient could explain why they attended the clinic, in the appropriate allocated space on the form. If unable to understand or answer the questions, the patient was assisted in completing the form by the clinic nurse. Assistance however, was rarely required.

The receptionist would then review the paperwork and direct the patient to either the doctor or nurse as indicated on the form. Patients were also asked to complete a satisfaction survey that enquired if the waiting time was acceptable and were asked their opinion on their consultation and treatment. 


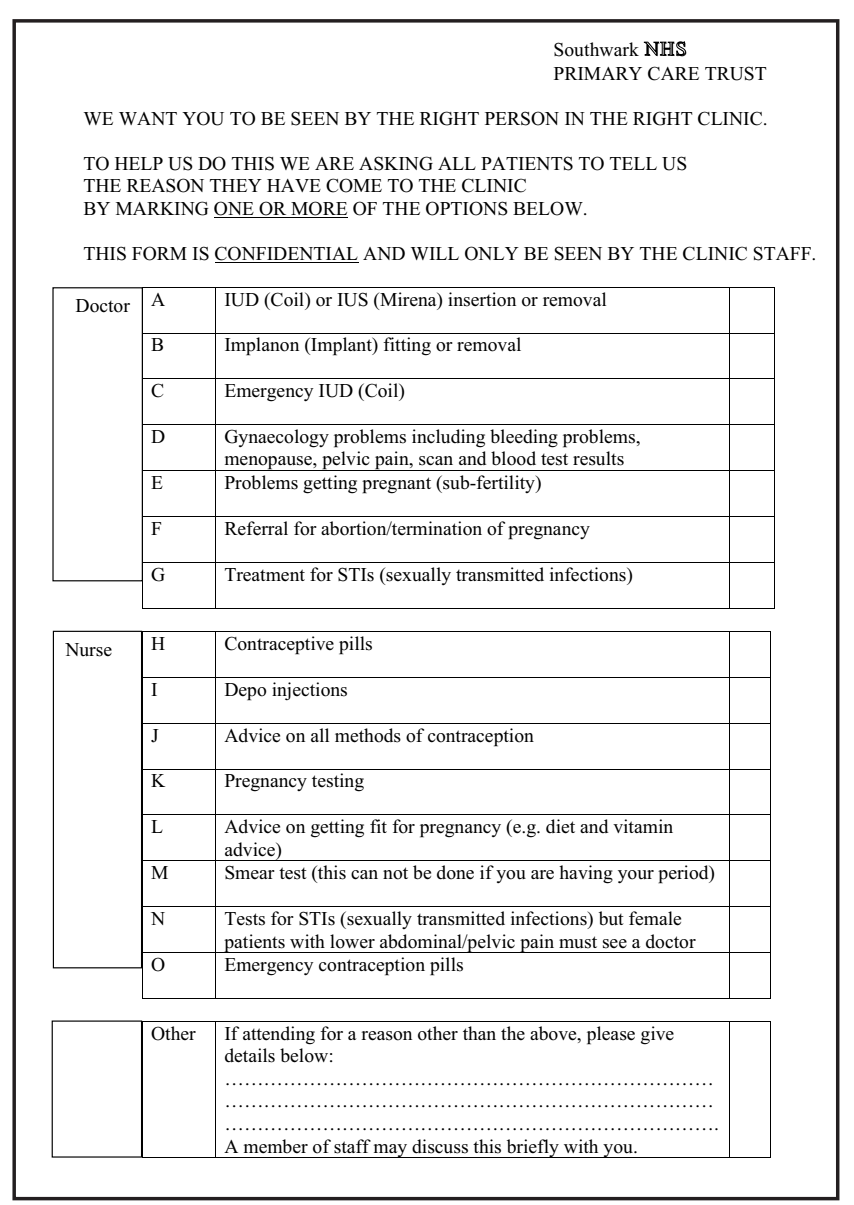

Figure 2 Example of triage form used in the pilot study

\section{Assessment of clinic processes}

The time of patient registration, when the doctor or nurse started and finished their consultation and when the patient left the clinic were recorded. This allowed for the calculation of the waiting time, the time spent with a clinician (treatment time) and the total time in the clinic. The number of clinicians seen by the patient was also documented.

\section{Assessment of the triage form}

The triage form was sent by the Southwark Primary Care Trust's (PCT) Public Involvement Team to a readers' panel for assessment of its usability. This panel comprises members of the public who live in the locality and who have volunteered to comment on written information provided by the PCT. This panel was asked for their opinion on the readability and ease of comprehension of the triage form. They were also asked to assess the ease-of-use of the options on the form and asked if they had any concerns about patients completing the form in a reception area.

\section{Ethical approval}

Ethical approval was not deemed necessary by the King's
College Hospital Research Ethics Committee as this study was classed as a service evaluation project.

\section{Statistical analysis}

Waiting time data were skewed (because a few patients waited much longer than the majority) and thus nonparametric tests were employed. The medians and interquartile ranges are presented pre- and post-triage system and the results were compared using the Wilcoxon rank-sum test. We estimated confidence intervals for generalised Hodges-Lehmann median differences in order to estimate the 'treatment effect' of triaging. The analysis was conducted using Stata for Windows 9.0 ${ }^{\text {TM }} 9$. Nominal variables (such as the proportion of patients requiring two clinicians) pre- and post-triage were compared using Chisquare tests. All the $p$ values are two-tailed.

\section{Results}

Data from 193 patients were collected; two self-assessment triage forms were incomplete and were not used in the analysis. Ninety-six patients were processed according to the traditional system and 97 patients filled in the selfassessment triage forms.

Comparison of the data showed that the treatment time was largely unaltered $11(7,19)$ minutes [results expressed as median (interquartile range)] with the traditional method vs $14(7,20)$ minutes with the triage method $(p=0.40)$ (Table 1). Conversely, the total time spent in the clinic was reduced from $58(34,75)$ minutes with the traditional method to $37(24,60)$ minutes with the triage system $(p=$ $0.0006)$. This was as a result of the significant reduction in waiting time from $40(22,60)$ to $23(10,40)$ minutes $(p<0.0001)$ (Table 1). Before the triage system was introduced, $20(21 \%)$ patients saw two clinicians on the same visit, whereas only $13(13 \%)$ did so after selfassessment was introduced $(p=0.17)$ (Table 1).

\section{Patient survey}

Thirty-seven (39\%) traditional process patients completed the satisfaction survey as did $32(33 \%)$ triage system patients. The introduction of self-triage did not alter patient satisfaction with the level of care, with $94.6 \%$ of the traditional process patients agreeing or strongly agreeing that their care was satisfactory vs $96.9 \%$ of patients experiencing the triage system $(p=0.64)$ (Table 2$)$. Self-triage did not alter

Table 2 Patient satisfaction survey before and after introduction of the triage system

\begin{tabular}{llll}
\hline Parameter & $\begin{array}{l}\text { Traditional } \\
\text { system } \\
(\boldsymbol{n}=37) \\
{[n,(\%)}\end{array}$ & $\begin{array}{l}\text { Triage } \\
\text { system } \\
(\boldsymbol{n}=37) \\
{[\boldsymbol{n},(\%)]}\end{array}$ & $\boldsymbol{p}$ \\
\hline $\begin{array}{l}\text { Patient satisfied with care } \\
\begin{array}{l}\text { (agree/strongly agree) } \\
\text { Waiting time acceptable }\end{array}\end{array}$ & $35(95)$ & $31(97)$ & 0.64 \\
(agree/strongly agree) & $31(84)$ & $26(81)$ & 0.78 \\
\hline
\end{tabular}

Table 1 Process and waiting times and number of patients seeing two clinicians on the same visit

\begin{tabular}{lllllr}
\hline Parameter & $\begin{array}{l}\text { Traditional system } \\
\text { [median (IQR)] }\end{array}$ & $\begin{array}{l}\text { Triage system } \\
\text { [median (IQR)] }\end{array}$ & $\boldsymbol{p}$ & $\begin{array}{l}\text { Median } \\
\text { difference }\end{array}$ & 95\% Cl \\
\hline Waiting time (minutes) & $40(22,60)$ & $23(10,40)$ & $<0.0001$ & 15 & 8,21 \\
Treatment time (minutes) & $11(7,19)$ & $14(7,20)$ & 0.40 & -1 & $-3,2$ \\
Total time (minutes) & $58(34,75)$ & $37(24,60)$ & 0.0006 & 15 & 7,23 \\
Patients seeing two clinicians [n, (\%)] & $20(21)$ & $13(13)$ & 0.17 & & \\
\hline
\end{tabular}

$\mathrm{Cl}$, confidence interval; IQR, interquartile range. 
the acceptability of waiting time: $83.7 \%$ patients thought that the waiting time was acceptable with the traditional system compared to $81.3 \%$ with the triage system $(p=0.78)$.

Thirty-six people completed the readers' panel survey. Almost $78 \%$ of respondents reported that the form was easy to understand, with $81 \%$ indicating that they found it simple to decide on which option to use when choosing the reason for their attendance. Two members of the panel found it difficult to differentiate between different options. It was also suggested that some of the acronyms could have been explained in more detail with more options for male patients included. The panel also felt that the form should have been available in different languages.

Although almost $81 \%$ of the readers' panel had no concerns about the form being completed at the reception desk, some suggested that a private area should be provided for completing the form. A number of panel members (39\%) expressed concern about confidentiality, although no patients raised this issue on the satisfaction survey.

\section{Discussion and conclusions}

This pilot study report is the first to demonstrate that a selfassessment triage form can lead to reductions in waiting times in SRH clinics. This achievement was largely due to less duplication of work by the nurse and doctor, with patients seeing the doctor directly if this was thought most appropriate. The traditional system is widely employed in clinics in the UK and therefore a slight alteration in practice may provide significant benefit. This self-triage process may also be transferable to other services where patients can be separated into different fast-track queues to appropriate specialist practitioners.

The triage system may deliver its greatest benefit to very busy clinics. If some patients need to be turned away as demand exceeds capacity, then those with urgent clinical problems can be prioritised, thus allowing alternative arrangements to be made for those with less urgent needs. In addition, the triage system can help ensure that patients waiting for specific procedures are not left to the end of the clinic where time might be too short (eg, for IUD insertions) or overruns occur if there are procedural complications. The overall efficiency of the clinic can therefore be improved with less inconvenience to patients.

Although the number of patients seeing two practitioners was reduced in our study, there was still a requirement for some patients to wait for the nurse and then the doctor. A more detailed triage form may help; however, there will always be patients who necessitate further review as the consultation evolves and more complex requirements become apparent. There needs to be a careful balance between the complexity and the ease of understanding of the form, and some patient crossover should be expected.

The exact division of labour between clinicians may also depend on the skill mix available in that clinic and the triage system will thus need to be flexible. For example, a nurse practitioner may fit IUDs and implants, while a less experienced nurse may only be able to prescribe according to a patient group directive.

A triage system may have implications for the Diploma of the Faculty of Sexual and Reproductive Healthcare (DFSRH) training, reducing doctor exposure to straightforward clients. This issue has increased over the years as the role of nurse practitioners has developed. Good training will necessitate senior nurses teaching DFSRH trainees, and doctors teaching nurse trainees. The DFSRH programme allows for this scenario. ${ }^{10}$

Interestingly, patient satisfaction did not appear to improve with the reduction of waiting time from a median of 40 to 23 minutes. This was probably due to a very high baseline satisfaction level. It may be that a larger study population or more sensitive questioning would allow us to find a real difference. Alternatively, it is possible that patients did not appreciate the 17 minute reduction in waiting time, perhaps as they had prepared themselves for a lengthy wait in a walk-in clinic as opposed to an appointment service. It may be that patients averse to long waits may have chosen to go elsewhere for their care, such as to a general practice surgery, where an appointment can be booked. We know that other factors such as the length of time spent with the patient ${ }^{11}$ and a clinician's communication skills ${ }^{12,13}$ may have greater influence on satisfaction levels.

We recognise that this study is limited by not having a parallel control group and that this may have allowed temporal-based bias. As a result, factors other than the triage system could have brought about a reduction in waiting times such as the time of year, case mix or changes in staffing. As this was a pilot study we did not collect demographic data such as patient age, sex or ethnic background, which might have accounted for some of the differences seen in waiting times between the two groups.

Waiting times are often skewed because if one or two complex patients take a long time to be seen then subsequent patients in the queue will (as a consequence) have a long wait.

Only one-third of patients completed the satisfaction survey. Although this is a reasonable response for this type of research, ${ }^{14}$ clearly this too could have introduced bias. Less-satisfied patients may not have completed the survey, for example. To address the readers' panel's concern over patient confidentiality when completing the assessment form we are investigating the use of election-style booths for form completion. Different language variations of the form are also being developed at the present time. These issues will need to be addressed by future research.

In conclusion, many SRH clinics have become extremely busy after the introduction of testing and treatment of genital infections. This can lead to longer waits for patients, which may lead to urgent patients leaving without being seen and can create a stressful working environment for clinic staff. Self-triage may reduce clinic waiting times and can help improve the organisation of a busy clinic.

\section{Acknowledgements}

The authors would like to thank Drs Helen Massil and Christine Robinson (Consultants in the Department of Sexual and Reproductive Health, Southwark Primary Care Trust, London) who supported this project.

\section{Statements on funding and competing interests} Funding None identified.

Competing interests None identified.

\section{References}

1 Department of Health. Choosing Health: Making Healthy Choices Easier. 2004. http://www.dh.gov.uk/en/Publications andstatistics/Publications/PublicationsPolicyAndGuidance/DH 4094550 [Accessed 14 June 2009].

2 Department of Health. Abortion Statistics, England and Wales: 2004. http://www.dh.gov.uk/PublicationsAndStatistics/ Publications/PublicationsStatistics/PublicationsStatisticsArticle /fs/en?CONTENT ID=4116461\&chk=6T9UTA [Accessed 14 June 2009].

3 Guys' and St Thomas' Charity Modernisation Initiative. http://www.gsttcharity.org.uk/grants/results_mi.html [Accessed 14 June 2009].

4 NHS Institute for Innovation and Improvement. Quality and Service Improvement Tools. http://www.nodelaysachiever.nhs. uk/Servicelmprovement/Tools/IT220_A+Comprehensive+Over view+of+Mapping+Processes+an $\bar{d}+$ Patient+Journeys.htm [Accessed 14 June 2009]. 
5 Modernisation Initiative - Sexual Health Services. http://www.mysexualhealth.org.uk/_data/assets/pdf_file/5927/ User_involvement_report.pdf [Accessed 14 June 2009].

6 Griffiths V, Ahmed-Jushuf IH. Is triage an appropriate way of dealing with walk-in patients attending genitourinary medicine clinics? Int J STD AIDS 2005; 16: 819-821.

7 Harindra V, Tobin JM, Tucker LJ. Triage clinics: a way forward in genitourinary medicine. Int J STD AIDS 2001; 12: 295-298.

8 Knight V, McNulty A. Triage in a public outpatient sexual health clinic. Sex Health 2006; 3: 87-90.

9 Newson R. Parameters behind "nonparametric" statistics: Kendall's tau, Somers' D and median differences. Stata Journal 2002; 2: 45-64.

10 Faculty of Sexual and Reproductive Healthcare. Training Requirements for Doctors Wishing to Obtain the Diploma of the Faculty of Sexual and Reproductive Healthcare (DFSRH). Form K/03 (07). http://www.ffprhc.org.uk/admin/uploads/
FormK.pdf [Accessed 14 June 2009].

11 Gross DA, Zyzanski SJ, Borawski EA, Cebul RD, Stange KC. Patient satisfaction with time spent with their physician. J Fam Pract 1998; 47: 133-137.

12 Shaw WS, Zaia A, Pransky G, Winters T, Patterson WB. Perceptions of provider communication and patient satisfaction for treatment of acute low back pain. J Occup Environ Med 2005; 47: 1036-1043.

13 Little P, Everitt H, Williamson I, Warner G, Moore M, Gould C, et al. Preferences of patients for patient centred approach to consultation in primary care: observational study. BMJ 2001; 322: 468-472.

14 Crow R, Gage H, Hampson S, Hart J, Kimber A, Storey L, et al. The measurement of satisfaction with healthcare: implications for practice from a systematic review of the literature. Health Technol Assess 2002; 6: 1-244.

\section{China blocks access to sex education websites}

China's government plans to tighten restrictions on websites that discuss sexual health as part of broader regulations. The Ministry of Health says the rules are aimed at improving the accuracy and scientific basis of all medical health sites on the Internet. The guidelines, which apply to topics including "sexual psychology, sexual ethics, sex medicine, and sex treatments", state that only governmentsanctioned medical institutions can provide sex-related content on websites, and that all such sites will have to be approved by provincial-level health authorities. Sex is a taboo subject in China, but rising incomes and increasing freedom of choice for how people behave have created more interest in sexual issues and in reproductive education. Many experts say education on the subject remains inadequate. Liang Peiding, who runs a site that offers information on issues such as sexual dysfunction and medicine, believes the health ministry shouldn't have the jurisdiction to monitor online content. Under the new restrictions, sex-related sites must use material only from specialists in the field. Violators can be fined up to 30000 yuan (about US\$4400).

Source: The Wall Street Journal, 26 June 2009

\section{USA birth rate mirrors recession}

The prospect of a 'baby-bust' with the current global recession mirrors the recessions of the 1930s and 1980s. Recent data from the USA census shows a strong slowdown in the birth rate that began in advance of the economic crisis. The figures have given rise to speculation that families anticipated hard times by having fewer children.

"If prospects look worse for families, they're going to be very likely to have fewer kids", said Mark Mather of the Population Research Bureau. The National Center for Health Statistics, which compiled the figures, said that it was too early to be sure why there were fewer pregnancies, but cited the economic slowdown and high house prices.

Source: http://news.bbc.co.uk/1/hi/business/8057326.stm

\section{Study stopped due to increased teen pregnancy rate}

A multi-million pound project aimed at improving health outcomes in teenagers has been cancelled after initial reports showed that it was not reducing teenage pregnancy rates. The intervention group $(n=2724)$ aged $13-15$ years at baseline deemed by professionals as at risk of teenage pregnancy, substance misuse, or school exclusion or to be vulnerable were offered support and advice from the Young People's Development Project. The prospective matched comparison study was to evaluate the effectiveness of the intervention in terms of drug and alcohol use and teenage conception rates The team from University College London report that the intervention group had higher rates of teenage pregnancy than the comparison group. ${ }^{1}$

\section{Reference}

Wiggins M, Bonell C, Sawtell M, Austerberry H Burchett $H$, Allen E, et al. Health outcomes of youth development programme in England: prospective matched comparison study. BMJ 2009; 339: b2534.

\section{Face of Global Sex Report}

Durex have published a report entitled Sex and Relationships Education: Assessing the Gaps for Eastern European Youth, that examines which variables are most likely to influence the perceived need for further sex and relationships education among 14-22-year-olds in Croatia, the Czech Republic, Hungary, Poland, Romania, Serbia and Slovakia.

The study shows that regardless of thei nationality, the younger a person is when they initially receive sex and relationships education, the less likely it is that they will feel the need for further sex education at later stages in their lives, particularly if the education is delivered by school teachers or health care professionals. The report concludes that preventative programmes in Eastern Europe should be formalised with universal access to children under the age of 12 years.

Source: http://www.durexnetwork.org/en-GB/research/faceof globalsex/Pages/Home.aspx

\section{Parents happy for teenage children} to carry contraception

A poll commissioned as part of the government's 'Time to Talk' campaign has shown that parents are no longer concerned about their children carrying contraception, with a fifth saying they have bought condoms to keep at home. The poll of more than 1000 people found that parents are no longer prudish about talking about sex. Just $4 \%$ of parents questioned said they would be anxious if they discovered their teenager, aged 16-19 years, was carrying contraception, with nine in ten saying they would want to talk to them about it. In addition, almost a quarter of parents (24\%) reported making an appointment with their local GP for their teenager to talk through the different types of contraception.

\section{Maternal BV and vitamin D levels}

Bacterial vaginosis (BV) is widespread and is associated with adverse pregnancy outcomes. A study investigating possible links with vitamin D status in pregnancy and BV in the first trimester of pregnancy found that BV declined as vitamin D levels rose, reaching a plateau at vitamin D levels of $80 \mathrm{nmol} / \mathrm{l}$. Vitamin D deficiency is associated with BV, possibly via the immune system. ${ }^{1}$ Vitamin D deficiency is associated with $\mathrm{BV}$ and may contribute to the strong racial disparity in the prevalence of $\mathrm{BV}$.

\section{Reference}

Bodnar LM, Krohn MA, Simhan HN. Maternal vitamin $D$ deficiency is associated with bacterial vaginosis in the first trimester of pregnancy. J Nutr 2009; 139 $1157-1161$.

\section{VF educational microsite}

The first interactive educational microsite for fertility and reproductive specialists has been launched at CookARTLab.com. Sponsored by Cook Medical, the site includes a video tour of an assisted reproductive technology (ART) laboratory - guiding the user through the whole in vitro fertilisation (IVF) process from ovum collection to embryo transfer. It also includes a knowledge base, discussion forum and news centre, all of which give ART specialists the opportunity to exchange knowledge and experience all in one place. This site gives an introduction into the processes involved in IVF and embryo transfer, which is of use for clinicians who need to discuss techniques with patients or who are planning to work in an IVF unit.

Source: http://CookARTLab.com

\section{Men having unprotected sex on} 'male pill'

A survey of 2000 people suggests that $64 \%$ of men would be more likely to have sex without a condom if they had taken the new male contraceptive injection. The survey also showed that over two-thirds of the men believed that they would have the new contraceptive injection. This rises amongst older men, with three-quarters of those over the age of 34 years likely to have the injection. Meanwhile, three in four women said they would not trust men to use the new contraceptive - and this lack of faith increases as women get older, with younger women more inclined to trust men on this issue.

Source: www.hall-and-partners.com

Reviewed by Henrietta Hughes, MRCGP, DFSRH General Practitioner, London, UK 